\title{
SEPARATION OF METEOR STREAMS FROM THE SPORADIC BACKGROUND
}

\author{
T.J. Jopek \\ Obserwatorium Astronomiczne UAM, \\ Ul.Słoneczna 36, 60-286 Poznań, Poland
}

\begin{abstract}
A cluster analysis procedure has been used to estimate the fraction of the sporadic interlopers (sporadis bias) identified as stream members among the observed meteor orbits. Using the artificial meteor orbits with the same distribution as the observed one, the sporadic bias is estimated for the given threshold value of the orbital similarity $D_{c}$. It has been shown that in case of the radio meteor catalogues the $D_{c}$ values given by the formula proposed in Southworth and Hawkins (1963)and in Lindblad (1971) correspond to the sporadic bias of 8-21\%. For the five radio meteor catalogues the values of $D_{c}$ corresponding to the fixed bias equal to $10 \%$ and $15 \%$ are given.
\end{abstract}

Key words: Meteors, D-criterion, orbital similarity threshold level.

\section{Introduction}

Unfortunately, every meteor stream searching procedure is biased by the sporadic component of the meteoroid population. Degree of this influence, among others, depends on the adopted threshold of the orbital similarity.

To estimate the similarity threshold $D_{c}$ between two meteor orbits one can use a rule given by Southworth and Hawkins (1963) or its modification by Lindblad (1971):

$$
\begin{gathered}
D_{c}=0.2\left(\frac{360}{N}\right)^{1 / 4}, \\
D_{c}=0.8(N)^{1 / 4},
\end{gathered}
$$

where $\mathrm{N}$ is the number of orbits. Both formulas are only an approximation, formula (1) was established on the basis of the small photographic meteor sample, formula (2) is a good working rule giving a little smaller values of $D_{c}$.

The threshold $D_{c}$ is the most important parameter of any stream searching procedure. It is strongly related with the infiltration of the sporadic background into results of the meteor streams identification. In the folowing sections we present some results of the studies of this relation.

Earth, Moon, and Planets 68: 339-346, 1995.

(C) 1995 Kluwer Academic Publishers. Printed in the Netherlands. 
TABLE I

The list of the utilized catalogues of the meteor orbital data. All orbits suspected to be outliers or having eccentricities $e \geq 1.5$ have been rejected. In fourth column the percentage of the meteor orbits with the radiant latitude $\beta_{R}>0$ is given.

\begin{tabular}{|l|r|r|r|r|}
\hline \multicolumn{1}{|c|}{$\begin{array}{c}\text { Meteor } \\
\text { catalogue }\end{array}$} & $\begin{array}{c}\text { Number } \\
\text { of orbits }\end{array}$ & $\begin{array}{c}\text { Used } \\
\text { orbits }\end{array}$ & $\begin{array}{c}\beta_{R}>0 \\
\%\end{array}$ & \multicolumn{1}{c|}{$\begin{array}{c}\text { Lim. } \\
\text { mag. }\end{array}$} \\
\hline Harvard 61-65 & 19327 & 19171 & 79.2 & $+12-13$ \\
Harvard 68-69 & 19818 & 19587 & 84.5 & $+12-13$ \\
Adelaide 60-61 & 2092 & 2092 & 38.8 & $+6-8$ \\
Obninsk 67-68 & 9358 & 9309 & 99.6 & $+6-8$ \\
Mogadisho 69-70 & 5328 & 5314 & 21.4 & $+6-8$ \\
Kharkov 1975 & 5317 & 5285 & 81.6 & $+12-13$ \\
KPOM-3 (phot.) & 1932 & 1906 & 77.5 & +3 \\
\hline
\end{tabular}

\section{Applied meteor orbital catalogues}

We have utilized five radio and one photographic meteor catalogue listed in Table I. All radio data origin from the IAU Meteor Data Centre (Lindblad $(1987,1991)$, Lindblad and Steel (1993)), however, only orbits without the error flag and the eccentricities $e<1.5$ has been used. The photographic catalogue KPOM-3 was used for comparison purposes. Its 1932 orbits are our own compilation of meteor data gathered from the literature (Jopek $(1989,1991)$ ).

Due to several reasons the orbital samples in Table I are not statistically equivalent, first of all due to different observational sites and schedule time. As an example, Fig. 1 shows the distributions of the perihelion distances and arguments of perihelions of the radio meteors observed in Kharkov and Mogadisho. Significant differences between these distributions are seen easily. Applying to the Kharkov and Mogadisho catalogues formulae (1) and (2) one will obtain almost the same values of the cut-off level, namely $D_{c}=0.1021$ and $D_{c}=0.1020$, respectively. Are these values adequate when applied for the meteor streams searching among these orbits? To give the answer to this question some numerical studies based on the cluster analysis in the equivalent sets of artificial meteor orbits has been performed.

\section{Generator of artificial meteor orbits}

Observed distribution of the meteor orbits is not the outcome of the stochastic process only. On Fig. 1 we have two examples of a very strong influence of the the Earth crossing condition on the distribution of the perihelion distances $q$ and 

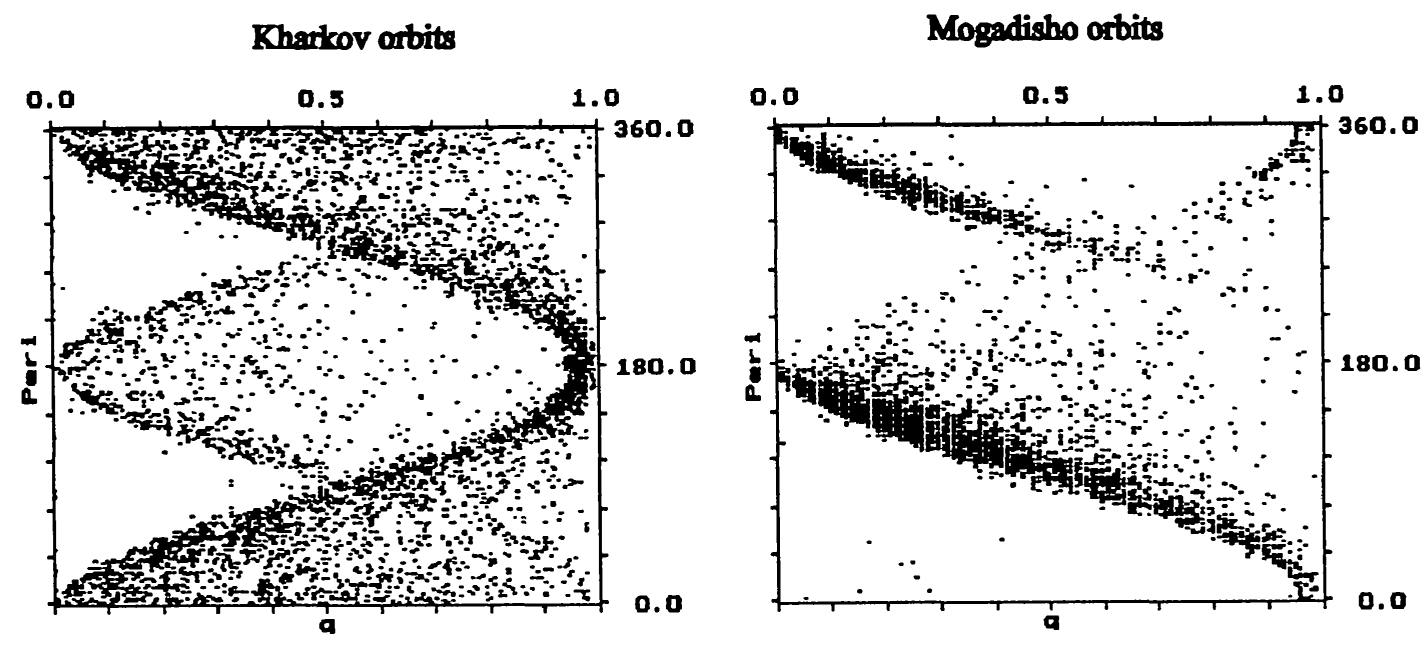

Fig. 1. Distributions of the perihelion distances and perihelion arguments of the Kharkov and Mogadisho catalogues. One can see strong influences of the observational selection effect caused by the Earth crossing condition and the location of the observational station.

perihelion arguments $\omega$. The crossing condition has the form:

$$
r=\frac{q(1+e)}{1 \pm e \cos \omega}
$$

where $q^{*}$ - the perihelion distance, e - the eccentricity, $\omega$ - the argument of perihelion, $\mathrm{r}$ - the heliocentric distance of the meteoroid $(r \in(0.983-1.017))$. The sign "-" should be applied for the ecliptic latitude of the meteor radiant $\beta_{R}>0$.

The artificial meteor orbits were drawn at random by the same algorithm as described in a paper Jopek, Froeschle (1994), namely:

- first, construct the observed distributions of $r, \beta_{R}, \Omega, i, e, \omega$,

- using observed distributions generate the random values of $r, \beta_{R}, \Omega, i, e, \omega$,

- having $r, \beta_{R}, e, \omega$ calculate the perihelion distance $\mathrm{q}$.

Obtained set of the artificial Earth crossing orbits has the same (statistically) distributions of the orbital elements as the observed sample and does not contain the associations in the genetic sense.

\section{Meteor streams searching procedure}

The streams searching procedure includes the association test between two orbits and the group searching algorithm. As the association test we have applied the 
orbital similarity function given by formula (4) (Jopek (1993)). This function is a hybrid of the functions proposed earlier by Southworth and Hawkins (1963) and by Drummond (1981):

$$
D_{H}^{2}=\left(e_{1}-e_{2}\right)^{2}+\left(\frac{q_{1}-q_{2}}{q_{1}+q_{2}}\right)^{2}+\left(2 \sin \frac{I_{12}}{2}\right)^{2}+\left(\frac{e_{1}+e_{2}}{2}\right)^{2}\left(2 \sin \frac{\pi_{12}}{2}\right)^{2},
$$

where where $I_{12}$ is the angle between the orbital planes and $\pi_{12}$ is the difference between the longitudes of perihelia measured from the common node of the orbits:

$$
\begin{aligned}
& {\left[2 \sin \frac{I_{12}}{2}\right]^{2}=\left[2 \sin \frac{i_{1}-i_{2}}{2}\right]^{2}+\sin i_{1} \sin i_{2}\left[2 \sin \frac{\Omega_{1}-\Omega_{2}}{2}\right]^{2},} \\
& \pi_{12}=\omega_{1}-\omega_{2}+2 \arcsin \left[\cos \frac{i_{1}+i_{2}}{2} \cdot \sin \frac{\Omega_{1}-\Omega_{2}}{2} \cdot \sec \frac{I_{12}}{2}\right]^{2},
\end{aligned}
$$

where the sign of the arcsin should be oposite when $\left|\Omega_{1}-\Omega_{2}\right|>180^{\circ}$.

The stream searching algorithm was implemented in a form of the computer routine. We have adopted the algorithm (a single neighbour linking technique) employed by Southworth and Hawkins (1963). There is a simple illustration of the run of this routine, namely, one may compare it with the creation - in the conceptual space of the orbital elements - a net of nodes. Each node represents a single orbit. Every two nodes one can conect by a section, the length of which represents degree of the similarity between two nodes (orbits). Hovewer only nodes remoted less then adopted value of the threshold $D_{c}$ are conected. When all pairs are checked, resulting conected nodes (subnets) represent the groups of associated orbits - the identified meteor streams.

Table II shows an example of the results of the cluster analysis among 5000 artificial orbits. Results obtained from the several of such tables give the possibility of averaging the parameters we are interested in. Following the idea from Krawczyk and Jopek (1993), in frame of the present paper we paid attention only to the mean percentage $S_{\%}$ of the orbits associated with the given $D_{c}$. This parameter we interpret as a general measure of the infiltration of the sporadic interlopers (sporadic bias) among the results obtained with the observed orbits.

\section{Results}

Fig. 2 illustrates changes of the sporadic bias $S_{\%}$ with the cut-off $D_{c}$ for the observed and artificial orbits. One can see significant differences between the curves. These curves have important practical applications, namely, one can use them to find the sporadic bias which fits to the given threshold level, particularly threshold given by formulae (1) or (2). For example, for the sample size of 1000 orbits, formula (1) gives $D_{c}=0.155$, and from the plot of the results obtained among 
TABLE II

Results of the cluster analysis among 5000 artificial orbits taken at random using the distribution of the orbital elements of the Harvard 68-69 catalogue. $D_{c}$ - the cutoff level, $S_{i \%}$ - the total percentage of the associated orbits, $S_{M}$ the number of groups with $\mathrm{M}$ members.

\begin{tabular}{|r|r|r|r|r|r|r|r|r|r|}
\hline$D_{c}$ & $S_{i \%}$ & $S_{2}$ & $S_{3}$ & $S_{4}$ & $S_{5}$ & $S_{6}$ & $S_{7}$ & $S_{8}$ & $\ldots$ \\
\hline$\ldots$ & $\ldots$ & $\ldots$ & $\ldots$ & $\ldots$ & $\ldots$ & $\ldots$ & $\ldots$ & $\ldots$ & $\ldots$ \\
0.080 & 12.1 & 219 & 25 & 8 & 3 & 0 & 1 & 0 & $\ldots$ \\
0.090 & 15.9 & 249 & 41 & 16 & 7 & 2 & 1 & 1 & $\ldots$ \\
0.100 & 20.8 & 297 & 66 & 17 & 9 & 8 & 3 & 1 & $\ldots$ \\
0.110 & 25.7 & 319 & 83 & 24 & 15 & 9 & 3 & 4 & $\ldots$ \\
0.120 & 31.2 & 344 & 95 & 33 & 15 & 11 & 11 & 5 & $\ldots$ \\
$\ldots$ & $\ldots$ & $\ldots$ & $\ldots$ & $\ldots$ & $\ldots$ & $\ldots$ & $\ldots$ & $\ldots$ & $\ldots$ \\
\hline
\end{tabular}

the artificial Adelaide data we have the corresponding bias $S_{\%}=18 \%$. In Table III (second and third column) one can see that the number of sporadic interlopers among the stream members identified with the thresholds obtained by formulae (1) and (2) is not constant. In case of formula (1) for the sample size of 1000 orbits $S \%$ varies in the interval $10-21 \%$. When formula (2) is employed the corresponding changes are $7.5-21 \%$.

To obtain results loaded by the same number of sporadic interlopers, independently on the origin of the orbital sample, its size, observational site, etc. we have to use different values of $D_{c}$, and they can be found also from Fig. 2. In the last column of Table III the values of $D_{c}$ corresponding to the fixed bias $S_{\%}=15 \%$ are given.

Performing similar studies for several samples of various sizes we can obtain the dependency of the cut-off level versus sample size for the fixed value of $S_{\%}$. On Fig. 3 we can single out three groups of orbital catalogues: (1) both Harvards and Kharkov catalogues, (2) the Obninsk and Adelaide catalogues and (3) the last group, the Mogadisho catalogue. In extent of each group the curves on Fig. 3 show some properties, namely:

(1) - for the sample sizes up to 3000 , obtained results among the Kharkov orbits resemble results obtained among the Harvard 60-61 catalogue. For the bigger samples they are more similar to the results obtained among the Harvard "Synoptic year" data. For the "Synoptic year" data from Fig. 3 we have that the constant percentage of the sporadic interlopers $S_{\%}=15 \%$ and $S_{\%}=10 \%$ correspond to the cut-off levels given by formula (1) and (2), respectively.

(2) - for Adelaide and Obninsk orbits the bias $S_{\%}=15 \%$ is obtained taking thresholds $D_{c}$ calculated by formula (2). 

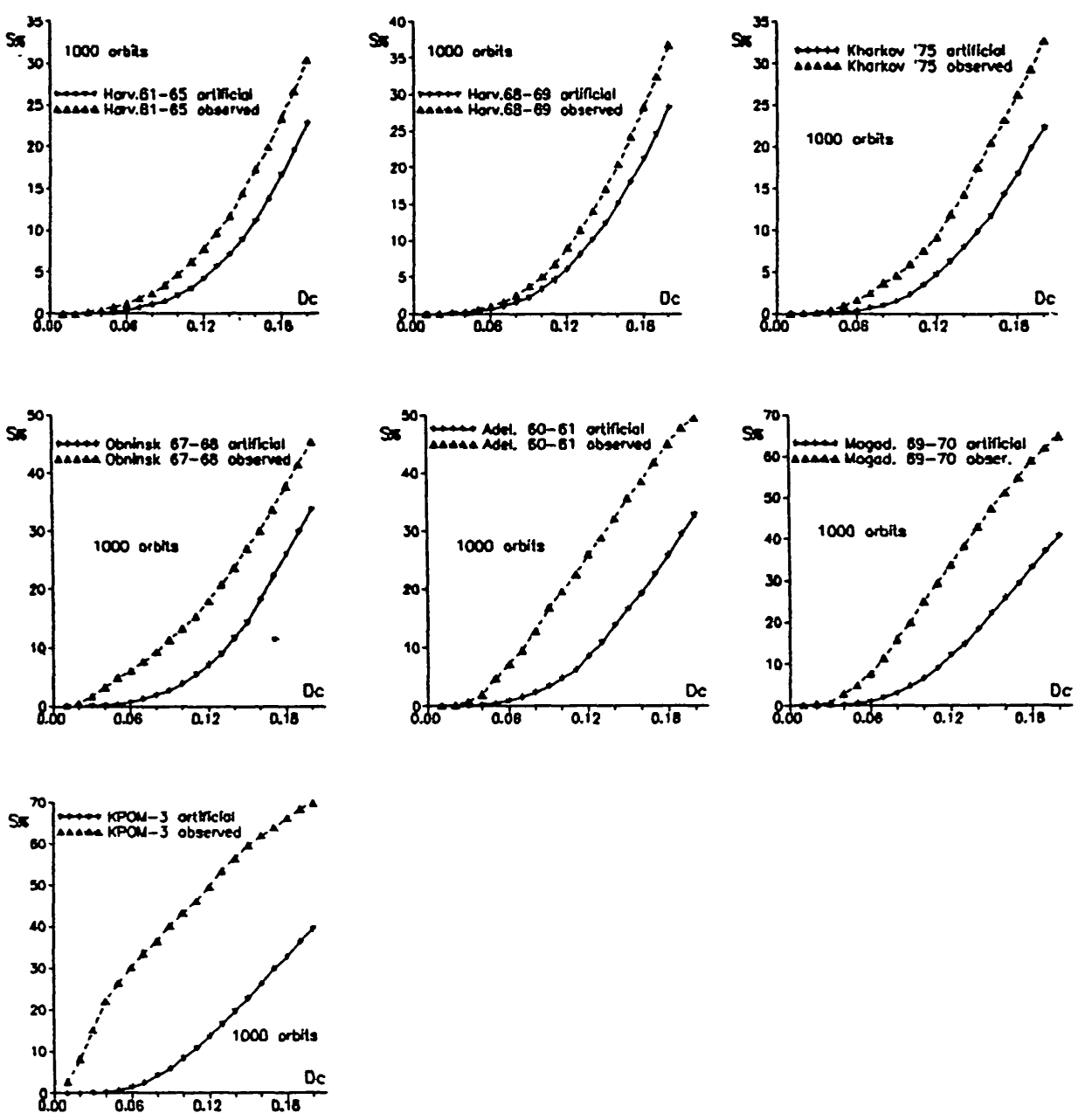

Fig. 2. The mean percentage $S_{\%}$ of the associated orbits versus threshold level $D_{c}$ of the orbital similarity.

(3) - in case of the Mogadisho catalogue, neither formula (1) nor formula (2) can be used for the sporadic bias level $S_{\%}=10 \%$ or $S_{\%}=15 \%$.

In Table IV we have gathetred the threshold values which fit to the constant sporadic bias $10 \%$ and $15 \%$ for the full size of the employed radio meteor catalogues. Now, we can return to the question asked in the second section of this paper, related with the meteor stream searching among the Kharkov and Mogadisho data. From Table IV it follows, that despite almost the same number of orbits, one has to apply different values of the threshold $D_{c}$ for these catalogues. On the other hand it is interesting to see that for Obninsk and Mogadisho orbits we have the same value of $D_{c}$, thought their sizes differ significantly. 

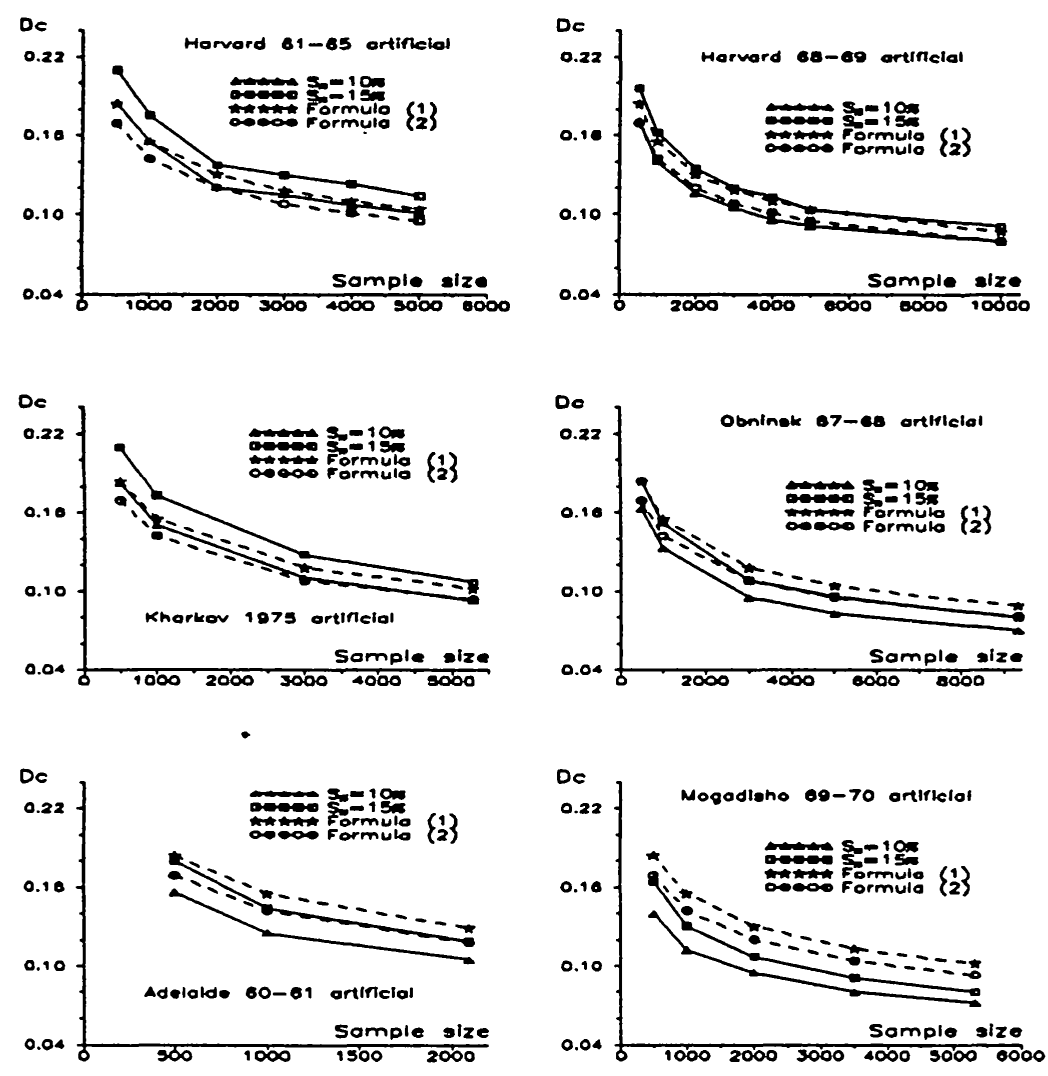

Fig. 3. The threshold levels versus orbital sample size. The solid curves correspond to the sporadic bias of $10 \%$ and $15 \%$. The dashed curves are obtained by formula (1).

\section{TABLE III}

Estimation of the fraction of sporadic interlopers $S_{\%}$ in the stream component identified among 1000 orbits. In the second and third column the $S_{\%}$ correspond to $D_{c}=0.155$ and $D_{c}=$ 0.142 obtained by formulae (1) and (2), respectively. The last column contains the cut-off level corresponding to the fixed bias $S_{\%}=15 \%$.

\begin{tabular}{|l|r|r|l|}
\hline \multicolumn{1}{|c|}{$\begin{array}{c}\text { Meteor orbital } \\
\text { sample }\end{array}$} & \multicolumn{2}{|c|}{$S_{\%}$} & \multicolumn{1}{|c|}{$D_{c}$} \\
\hline Harvard 61-65 & 10 & 7.5 & 0.175 \\
Harvard 68-69 & 13 & 11 & 0.16 \\
Adelaide 60-61 & 18 & 15 & 0.14 \\
Obninsk 67-68 & 16 & 12 & 0.15 \\
Mogadisho 69-70 & 22 & 19 & 0.13 \\
Kharkov 1975 & 11 & 8 & 0.17 \\
KPOM-3 & 24 & 21 & 0.125 \\
\hline
\end{tabular}




\section{TABLE IV}

The thresholds $D_{c}$ of the orbital similarity for the selected radio meteor catalogues. The values of $D_{c}$ corresponds to $10 \%$ and $15 \%$ of the sporadic bias.

\begin{tabular}{|l|r|l|l|}
\hline \multirow{2}{*}{$\begin{array}{c}\text { Radio meteor } \\
\text { catalogue }\end{array}$} & \multirow{2}{*}{$\begin{array}{c}\text { Sample } \\
\text { size }\end{array}$} & \multicolumn{2}{|c|}{$D_{c}$} \\
\cline { 3 - 4 } & & $S_{\%}=10$ & $S_{\%}=15$ \\
\hline Harvard 61-65 & 19171 & 0.72 & 0.83 \\
Harvard 68-69 & 19587 & 0.065 & 0.075 \\
Adelaide 60-61 & 2092 & 0.105 & 0.119 \\
Obninsk 67-68 & 9309 & 0.07 & 0.08 \\
Mogadisho 69-70 & 5314 & 0.072 & 0.08 \\
Kharkov 1975 & 5285 & 0.093 & 0.107 \\
\hline
\end{tabular}

\section{References}

Drummond J.D.: 1981,'A test of Comet and meteor shower associations', ICARUS 45, pp. 545553.

Jopek T.J.: 1989,'Catalogue of precise meteor orbits', Astron.Obs. UAM Tech.Report 6/89 CPBP 01.11 , (in polish)

Jopek T.J.: 1991,'Catalogue of precise meteor orbits', in Proceedings of the Conference on Dynamic of Small Bodies of the Solar System, Polish-Slovak Conference, Warsaw, October 25-28, 1988 (Ziolkowski $K ., E d$. ), pp.49-55, (in polish)

Jopek T.J.: 1993,'Remarks on the Meteor Orbital Similarity D-Criterion', ICARUS 106, pp. 603-607.

Jopek T.J., Froeschle C.: 1994,(in preparation for Astronomy and Astrophysics)

Krawczyk A., Jopek T.J.: 1993,'Separation of meteor streams from the sporadic background', in Proc. Conf. on Dynamics and Astrometry of Natural and Artificial Celestial Bodies. Astronomical Observatory Poznań, Poland, September 1993, (K.Kurzyńska, F.Barlier,P.K.Seidelmann,I.Wytrzyszczak, eds.), pp. 227-230

Lindblad B.A.: 1971,'A computerized stream search among 865 precise photographic meteor orbits', Smithson. Contrib.Astrophys. 12, pp. 1-12

Lindblad B.A.: 1987, in Interplanetary Matter, Proc. 10th European Reg. Meeting of the IAU, Prague, (Z.Ceplecha, P.Pecina eds.), 2, pp. 201-204.

Lindblad B.A.: 1991,in Origin and Evolution of Interplanetary Dust, (A.C.Levasseur-Regourd, Hasegawa H. eds.) Kluwer Acad. Publishing, pp. 311-314.

Lindblad B.A., Steel D.I.: 1993, 'Meteoroid orbits available from the IAU Meteor Data Center',in IAU Symp. 160: Asteroids, Comets, Meteors, (A.Milani, M. Di Martino and A.Celino eds.) Kluver Acad. Publishing, Dordrecht, Holland, pp. 497-501

Southworth R.B., Hawkins G.S.: 1963,'Statistics of meteor streams', Smithson.Contrib.Astrophys. 7, pp. $261-285$ 